\title{
Axial charges of octet and decuplet baryons
}

\author{
Ki-Seok Choi, W. Plessas, and R.F. Wagenbrunn \\ Theoretische Physik, Institut für Physik, Karl-Franzens-Universität, Universitätsplatz 5, A-8010 Graz, Austria
}

\begin{abstract}
We present a study of axial charges of baryon ground and resonant states with relativistic constituent quark models. In particular, the axial charges of octet and decuplet $N, \Sigma, \Xi, \Delta, \Sigma^{*}$, and $\Xi^{*}$ baryons are considered. The theoretical predictions are compared to existing experimental data and results from other approaches, notably from lattice quantum chromodynamics and chiral perturbation theory. The relevance of axial charges with regard to $\pi$-dressing and spontaneous chiral-symmetry breaking is discussed.
\end{abstract}

PACS numbers: 12.39.Ki, 14.20.Dh, 14.20.Jn

Keywords: Axial charge, Relativistic quark model, Baryon properties

\section{INTRODUCTION}

The axial charges $g_{A}$ of baryon states are essential quantities for the understanding of both the electroweak and strong interactions within the Standard Model of elementary-particle physics. They do not only govern weak processes, such as the $\beta$ decay, but also intertwine the weak and strong interactions. This is most clearly reflected by the Goldberger-Treiman relation, which in case of the $N$ reads $g_{A}=f_{\pi} g_{\pi N N} / M_{N}$ [1]. Given the $\pi$ decay constant $f_{\pi}$ and the nucleon mass $M_{N}$, the $\pi N N$ coupling constant $g_{\pi N N}$ just turns out to be proportional to $g_{A}$. Thus the relevance of $\pi$ degrees of freedom in (lowand intermediate-energy) hadronic physics is intimately tied to the axial charges: Whenever $g_{A}$ becomes sizable, $\pi$ degrees of freedom should matter sensibly. Therefore $g_{A}$ can also be viewed as an indicator of the phenomenon of spontaneous breaking of chiral symmetry $(\mathrm{SB} \chi \mathrm{S})$ of non-perturbative quantum chromodynamics (QCD), which is manifested by the non-vanishing value of the light-flavor chiral condensate $\langle 0|q \bar{q}| 0\rangle^{1 / 3} \approx-235$ $\mathrm{MeV}$. The axial charges thus constitute important parameters for low-energy effective theories. Any reasonable model of non-perturbative QCD should yield the $g_{A}$ of correct sizes. In fact, the axial charges may be considered as benchmark observables for the nucleon, and more comprehensively the baryon, structures.

Best known is, of course, the axial charge of the $N$, as its experimental value can be deduced from the ratio of the axial to the vector coupling constants $g_{A} / g_{V}=$ $1.2695 \pm 0.0029$ [2]; usually this is done under the assumption of conserved vector currents (CVC), which implies $g_{V}=1$. The deviation of $g_{A}$ from 1 , the axial charge of a point-like particle, can be attributed, according to the Adler-Weisberger sum rule [3, 4], to the differences between the $\pi^{+} N$ and $\pi^{-} N$ cross sections in pion-nucleon scattering. Unfortunately, axial charges of other baryon (ground) states are not known directly from experiment.

The axial charges are also 'measured' in lattice QCD. An increasing number of results has recently become available, even from full-QCD lattice calculations. A recent summary of the lattice-QCD results for the $g_{A}$ of the nucleon is presented in ref. [5]. The axial charges of hyperons have been studied by Lin et al. [6] as well as Erkol et al. 7] and Engel et al. [8] in (2+1)- and 2-flavor lattice $\mathrm{QCD}$, respectively. There have also been a number of other attempts to explain the axial charges of the $N$ and the other baryons. We mention only the more modern ones through chiral perturbation theory $(\chi \mathrm{PT})$ (see the recent review by Bernard [9] or, for example, ref. [10]), chiral unitary approaches [11], and relativistic constituent quark models (RCQM) 12 14].

Recently, also the axial charges of the $N$ resonances have come into the focus of interest, because of the question of restoration of chiral symmetry higher in the baryon (as well as meson) spectra. Specifically, it has been argued that the magnitudes of $g_{A}$ should become small for almost degenerate parity-partner $N$ resonances, indicating the onset of chiral-symmetry restoration with higher excitation energies [15, [16]. As the $g_{A}$ values of $N$ resonances are not known from phenomenology and can hardly be measured experimentally, this remains a highly theoretical question. However, the problem can be explored with the use of lattice QCD. Corresponding first results have already become available, but only for two of the $N$ resonances, namely, $N(1535)$ and $N(1650)$ [17]. Both of them have spin $J=\frac{1}{2}$ and parity $P=-1$. Since there is not yet any lattice-QCD result for positiveparity states, the above issue relating to parity-doubling remains unresolved from this side.

The problem of $g_{A}$ of the $N$ resonances has most recently also been studied within the RCQM [18]. The axial charges of all the $N$ resonances up to $\sim 1.9 \mathrm{GeV}$ and $J^{P}=\frac{1}{2}^{ \pm}, \frac{3}{2}^{ \pm}, \frac{5}{2}^{ \pm}$have been calculated with $N$ resonance wave functions stemming from realistic RCQM with Goldstone-boson-exchange (GBE) as well as onegluon-exchange (OGE) hyperfine interactions. One has found the remarkable result that, especially in case of the GBE RCQM, the magnitudes of the axial charges need not be small, even if the energy levels of the opposite-parity partners become (almost) degenerate at increased excitation energies, e.g. the $J^{P}=\frac{5}{2}^{ \pm}$resonances $N(1680)$ and $N(1675)$. Thus the issue of possible chiral-restoration phenomena reflected by the axial charges remains tantalizing until further insights become available (e.g. from lattice QCD or alternative attempts). 
Another question related to the axial charges of the $N$ resonances concerns the role of $\{Q Q Q Q \bar{Q}\}$ components. It has been argued that sizable admixtures of $\{Q Q Q Q \bar{Q}\}$ are needed in order to reproduce an almost vanishing $g_{A}$ of the $N(1535)$ resonance [19, 20]. However, these results are usually obtained in a simplistic non-relativistic approach. Meanwhile it is known that a RCQM with realistic $\{Q Q Q\}$ wave functions can easily explain a practically vanishing $g_{A}$ of $N(1535)$ [18], in perfect congruency with the predictions obtained from lattice QCD [17], and there is no need for considerable $\{Q Q Q Q \bar{Q}\}$ admixtures in this case. Moreover, the correct sizes of the axial charges of the $N$ ground state and the $N(1535)$ as well as $N(1650)$ resonances can simultaneously and consistently be reproduced within a RCQM with only $\{Q Q Q\}$ configurations [18].

In the context of hyperons the axial charges are also important to learn about the role of $S U(6)$ flavorsymmetry breaking. In particular, in the case of conserved $S U(3)_{F}$ the axial charges of the $N, \Sigma$, and $\Xi$ ground states are connected by the following simple relations [21, 22]

$$
g_{A}^{N}=F+D, \quad g_{A}^{\Sigma}=\sqrt{2} F, \quad g_{A}^{\Xi}=F-D,
$$

which follow through $S U(3)$ Clebsch-Gordan coefficients in the decomposition of the axial form factor into the functions $F$ and $D$ relating to the octet components in $S U(3)$ 23]. Note that we adopt the convention of $g_{A} / g_{V}$ being positive for the $N$ (like in ref. [21]) contrary to the PDG [2]; this then determines also the signs of all other baryon axial charges according to Eq. (11).

In the present paper we present results from a comprehensive study of the axial charges of octet and decuplet ground states $N, \Sigma, \Xi, \Delta, \Sigma^{*}$, and $\Xi^{*}$ as well as their resonances along RCQMs. In particular, we employ the RCQMs whose quark-quark hyperfine interactions derive from OGE [24] and GBE dynamics [25]; in the latter case we consider both the version with only the spin-spin interaction from pseudoscalar exchange (psGBE) [26] as well as the extended version that includes all force components (i.e. central, tensor, spin-spin, and spin-orbit) from pseudoscalar, scalar, and vector exchanges (EGBE) [27]. The calculations are performed in the framework of Poincarè-invariant quantum mechanics. In order to keep the numerical computations manageable, we have to restrict the axial current operator to the so-called spectator model (SM). It means that the weak-interaction gauge boson couples only to one of the constituent quarks in the baryon. This approximation has turned out to be very reasonable already in a previous study of the axial and induced pseudoscalar form factors of the nucleon [12], where the SM was employed specifically in the point form (PF) of relativistic quantum mechanics [28]. It has also been used in studies of the electromagnetic structure of the $N$, reproducing both the proton and neutron form factors in close agreement with the experimental data [13, 29 31].

In the following chapter we explain the formalism for the calculation of the matrix elements of the axial current operator and give the definition of the axial charges for the different baryon ground and resonant states. Subsequently we present the results and compare them to experimental data as well as to results from other approaches, notably from lattice $\mathrm{QCD}$ and $\chi P T$. In the final chapter we draw our conclusions.

\section{FORMALISM}

In hadronic physics the (diagonal) baryon axial charges $g_{A}^{B}$ govern such processes like $n \rightarrow p e^{-} \bar{\nu}_{e}, \Sigma^{-} \rightarrow \Sigma^{0} e^{-} \bar{\nu}_{e}$, $\Xi^{-} \rightarrow \Xi^{0} e^{-} \bar{\nu}_{e}$ etc. They can generally be calculated through semileptonic decays $B_{1} \rightarrow B_{2} \ell \bar{\nu}$ with strangeness change $\Delta S=0$. The axial charge is conveniently defined through the value of the axial form factor $G_{A}\left(Q^{2}\right)$ at $Q^{2}=0$, where $Q^{2}=-q^{2}$ is the fourmomentum transfer. The axial form factor $G_{A}\left(Q^{2}\right)$ can be deduced from the relativistically invariant matrix element of the axial current operator $\hat{A}_{+}^{\mu}\left(Q^{2}\right)$ sandwiched between the eigenstates of baryons $B_{1}$ and $B_{2}$. Here, the subscript + refers to the isospin-raising ladder operator $\tau_{+}=\frac{1}{2}\left(\tau_{1}+i \tau_{2}\right)$, with $\tau_{i}$ being the usual Pauli matrices. In the specific case of the neutron $\beta$ decay the matrix element of $\hat{A}_{+}^{\mu}\left(Q^{2}=0\right)$ reads

$$
\left\langle p\left|\hat{A}_{+}^{\mu}\right| n\right\rangle=g_{A}^{N} \bar{U}_{p}\left(P, J_{3}^{\prime}\right) \gamma^{\mu} \gamma_{5} \frac{\tau_{+}}{2} U_{n}\left(P, J_{3}\right),
$$

where $U_{n}$ and $U_{p}$ are the neutron and proton spinors, depending on the four-momentum $P$ and helicities $J_{3}$ and $J_{3}^{\prime}$, respectively; $\gamma^{\mu}$ and $\gamma_{5}$ are the usual Dirac matrices. Alternatively, the matrix element in Eq. (2) can also be expressed as

$$
\left\langle p\left|\hat{A}_{3}^{\mu}\right| p\right\rangle=g_{A}^{N} \bar{U}_{p}\left(P, J_{3}^{\prime}\right) \gamma^{\mu} \gamma_{5} \frac{\tau_{3}}{2} U_{p}\left(P, J_{3}\right)
$$

or

$$
\left\langle n\left|\hat{A}_{3}^{\mu}\right| n\right\rangle=g_{A}^{N} \bar{U}_{n}\left(P, J_{3}^{\prime}\right) \gamma^{\mu} \gamma_{5} \frac{\tau_{3}}{2} U_{n}\left(P, J_{3}\right) .
$$

In the spirit of the latter relations we may express the axial charge $g_{A}^{B}$ of any baryon $B=N, \Delta, \Sigma, \Xi, \ldots$ and its resonances more generally. Let us denote the baryon states by $\left|B ; P, J, J_{3}\right\rangle$, i.e. as simultaneous eigenstates of the four-momentum operator $\hat{P}^{\mu}$, the intrinsic-spin operator $\hat{J}$ and its $z$-projection $\hat{J}_{3}$. Since $\hat{P}^{\mu}$ and the invariant mass operator $\hat{M}$ commute, these eigenstates can be obtained by solving the eigenvalue equation of $\hat{M}$

$$
\hat{M}\left|B ; P, J, J_{3}\right\rangle=M\left|B ; P, J, J_{3}\right\rangle,
$$

Then the axial charge $g_{A}^{B}$ of any baryon state $B$ with $J=\frac{1}{2}, \frac{3}{2}, \frac{5}{2}$ is given by the matrix elements of the axial 
current operator $\hat{A}_{3}^{\mu}$ for zero momentum transfer $Q^{2}$ as

$$
\begin{gathered}
\left\langle B ; P, \frac{1}{2}, J_{3}^{\prime}\left|\hat{A}_{3}^{\mu}\right| B ; P, \frac{1}{2}, J_{3}\right\rangle= \\
C_{B} \bar{U}_{B}\left(P, J_{3}^{\prime}\right) g_{A}^{B} \gamma^{\mu} \gamma_{5} \frac{\tau_{3}}{2} U_{B}\left(P, J_{3}\right), \\
\left\langle B ; P, \frac{3}{2}, J_{3}^{\prime}\left|\hat{A}_{3}^{\mu}\right| B ; P, \frac{3}{2}, J_{3}\right\rangle= \\
C_{B} \bar{U}_{B}^{\nu}\left(P, J_{3}^{\prime}\right) g_{A}^{B} \gamma^{\mu} \gamma_{5} \frac{\tau_{3}}{2} U_{B ; \nu}\left(P, J_{3}\right), \\
\left\langle B ; P, \frac{5}{2}, J_{3}^{\prime}\left|\hat{A}_{3}^{\mu}\right| B ; P, \frac{5}{2}, J_{3}\right\rangle= \\
C_{B} \bar{U}_{B}^{\nu \lambda}\left(P, J_{3}^{\prime}\right) g_{A}^{B} \gamma^{\mu} \gamma_{5} \frac{\tau_{3}}{2} U_{B ; \nu \lambda}\left(P, J_{3}\right),
\end{gathered}
$$

where the coefficients $C_{B}$ are specified by

$$
C_{N}=2 C_{\Delta}=\frac{1}{\sqrt{2}} C_{\Sigma}=C_{\Xi}=1 .
$$

Here, $U_{B}\left(P, J_{3}\right)$ are the usual Dirac spinors for $J=\frac{1}{2}$ baryons and $U_{B ; \nu}\left(P, J_{3}\right)$ as well as $U_{B ; \nu \lambda}\left(P, J_{3}\right)$ are the Rarita-Schwinger spinors [32] for $J=\frac{3}{2}$ and $J=\frac{5}{2}$ baryons, respectively, with the normalizations as given in Appendix A.

Omitting from now on the denotation after $B$ we can write the matrix elements of $\hat{A}_{3}^{\mu}$ for any ground and resonance states as

$$
\begin{aligned}
& \left\langle P, J, J_{3}^{\prime}\left|\hat{A}_{3}^{\mu}\left(Q^{2}=0\right)\right| P, J, J_{3}\right\rangle= \\
& 2 M \sum_{\sigma_{i} \sigma_{i}^{\prime}} \int d^{3} \vec{k}_{1} d^{3} \vec{k}_{2} d^{3} \vec{k}_{3} \frac{\delta^{3}\left(\vec{k}_{1}+\vec{k}_{2}+\vec{k}_{3}\right)}{2 \omega_{1} 2 \omega_{2} 2 \omega_{3}} \\
& \times \Psi_{P J J_{3}^{\prime}}^{\star}\left(\vec{k}_{1}, \vec{k}_{2}, \vec{k}_{3} ; \sigma_{1}^{\prime}, \sigma_{2}^{\prime}, \sigma_{3}^{\prime}\right) \\
& \times\left\langle k_{1}, k_{2}, k_{3} ; \sigma_{1}^{\prime}, \sigma_{2}^{\prime}, \sigma_{3}^{\prime}\left|\hat{A}_{3}^{\mu}\right| k_{1}, k_{2}, k_{3} ; \sigma_{1}, \sigma_{2}, \sigma_{3}\right\rangle \\
& \times \Psi_{P J J_{3}}\left(\vec{k}_{1}, \vec{k}_{2}, \vec{k}_{3} ; \sigma_{1}, \sigma_{2}, \sigma_{3}\right) .
\end{aligned}
$$

The $\Psi$ 's are the momentum-space representations of the baryon eigenstates for $\vec{P}=0$, i.e. the rest-frame wave functions of the baryon ground and resonance states with corresponding mass $M$ and total angular momentum $J$ and $z$-projections $J_{3}$ as well as $J_{3}^{\prime}$. Here they are expressed as functions of the individual quark threemomenta $\vec{k}_{i}$, which sum up to $\vec{P}=\vec{k}_{1}+\vec{k}_{2}+\vec{k}_{3}=0$; $\omega_{i}=\sqrt{m_{i}^{2}+\vec{k}_{i}^{2}}$ is the energy of quark $i$ with mass $m_{i}$, and the individual-quark spin orientations are denoted by $\sigma_{i}$.

The integral on the r.h.s. of Eq. (8) is evaluated along the SM what amounts to the matrix element of the axial current operator $\hat{A}_{a}^{\mu}$ between (free) three-particle states $\left|k_{1}, k_{2}, k_{3} ; \sigma_{1}, \sigma_{2}, \sigma_{3}\right\rangle$ to be assumed in the form

$$
\begin{array}{r}
\left\langle k_{1}, k_{2}, k_{3} ; \sigma_{1}^{\prime}, \sigma_{2}^{\prime}, \sigma_{3}^{\prime}\left|\hat{A}_{3}^{\mu}\right| k_{1}, k_{2}, k_{3} ; \sigma_{1}, \sigma_{2}, \sigma_{3}\right\rangle= \\
3\left\langle k_{1}, \sigma_{1}^{\prime}\left|\hat{A}_{3, \mathrm{SM}}^{\mu}\right| k_{1}, \sigma_{1}\right\rangle 2 \omega_{2} 2 \omega_{3} \delta_{\sigma_{2} \sigma_{2}^{\prime}} \delta_{\sigma_{3} \sigma_{3}^{\prime}} .
\end{array}
$$

For point-like quarks this matrix element involves the axial current operator of the active quark 1 (with quarks 2 and 3 being the spectators) in the form

$$
\left\langle k_{1}, \sigma_{1}^{\prime}\left|\hat{A}_{3, \mathrm{SM}}^{\mu}\right| k_{1}, \sigma_{1}\right\rangle=\bar{u}\left(k_{1}, \sigma_{1}^{\prime}\right) g_{A}^{q} \gamma^{\mu} \gamma_{5} \frac{\tau_{3}}{2} u\left(k_{1}, \sigma_{1}\right),
$$

where $u\left(k_{1}, \sigma_{1}\right)$ is the spinor of a quark with flavor $u$ or $d$ and $g_{A}^{q}=1$ its axial charge. A pseudovector current analogous to the one in Eq. (10) was recently also used in the calculation of $g_{\pi N N}$ and the strong $\pi N N$ vertex form factor in ref. [33].

For the calculation of the axial charges $g_{A}$ we can use either one of the components $\mu=i=1,2,3$ of the axial current operator $\hat{A}_{3, \mathrm{SM}}^{\mu}$ in Eq. (10). The expression on the r.h.s. then specifies to

$$
\begin{aligned}
& \bar{u}\left(k_{1}, \sigma_{1}^{\prime}\right) \gamma^{i} \gamma_{5} \frac{\tau_{3}}{2} u\left(k_{1}, \sigma_{1}\right)= \\
& 2 \omega_{1} \chi_{\frac{1}{2}, \sigma_{1}^{\prime}}^{*}\left\{\left[1-\frac{2}{3}(1-\kappa)\right] \sigma^{i}\right. \\
& \left.+\sqrt{\frac{5}{3}} \frac{\kappa^{2}}{1+\kappa}\left[\left[\vec{v}_{1} \otimes \vec{v}_{1}\right]_{2} \otimes \vec{\sigma}\right]_{1}^{i}\right\} \frac{\tau_{3}}{2} \chi_{\frac{1}{2}, \sigma_{1}},
\end{aligned}
$$

where $\kappa=1 / \sqrt{1+v_{1}^{2}}$ and $\vec{v}_{1}=\vec{k}_{1} / m_{1}$. Herein $\sigma^{i}$ is the $i$-th component of the usual Pauli matrix $\vec{\sigma}$ and $v_{1}$ the magnitude of the three-velocity $\vec{v}_{1}$. The symbol $[\cdot \otimes \cdot]_{k}^{i}$ denotes the $i$-th component of a tensor product $[. \otimes \cdot]_{k}$ of rank $k$. We note that a similar formula was already published before by Dannbom et al. 22], however, restricted to the case of total orbital angular momentum $L=0$. Our expression holds for any $L$, thus allowing to calculate $g_{A}$ for the most general wave function of a baryon ground or resonances state specified by intrinsic spin and parity $J^{P}$.

\section{RESULTS}

In Table $\prod$ we present the RCQM results from our calculations for the axial charges $g_{A}^{B}$ of the octet and decuplet ground states $B=N, \Sigma, \Xi, \Delta, \Sigma^{*}$, and $\Xi^{*}$. Except for the $N$ there are no direct experimental data for $g_{A}$ (from $\Delta S=0$ decays). The predictions for $g_{A}^{N}$ by all three RCQMs come close to the experimental value, with all of them falling slightly below it. This is also the trend of present-day lattice-QCD calculations [5]; only the EOT result seems to represent a notable exception, even if we take the theoretical uncertainties into account (in Table — we have chosen to quote the EOT result corresponding to their calculation with the smallest quark mass of 35 $\mathrm{MeV}$ ). In addition, also the JT prediction obtained from $\chi \mathrm{PT}$ remains below the experimental value. There might be a variety of reasons why the different approaches underestimate the $g_{A}^{N}$. However, one should also bear in mind that the phenomenological value of $g_{A}^{N} \sim 1.27$ is supposed under the conjecture of conserved vector currents. What concerns the RCQMs, and in particular 
TABLE I: Axial charges $g_{A}^{B}$ of octet and decuplet ground states as predicted by the EGBE 27], psGBE [26], and OGE 24] RCQMs in comparison to experiment [2] and lattice QCD results from Lin and Orginos (LO) [6] and Erkol, Oka, and Takahashi (EOT) 7] as well as $\chi \mathrm{PT}$ results from Jiang and Tiburzi (JT) [10, 34]; also given is the nonrelativistic limit (NR) from the EGBE RCQM.

\begin{tabular}{|c|c|c|c|c|c|c|c|c|}
\hline & $\operatorname{Exp}$ & EGBE & psGBE & OGE & $\mathrm{LO}$ & EOT & JT & NR \\
\hline $\mathrm{N}$ & $1.2695 \pm 0.0029$ & 1.15 & 1.15 & 1.11 & $1.18 \pm 0.10$ & $1.314 \pm 0.024$ & 1.18 & 1.65 \\
\hline$\Sigma$ & - & 0.65 & 0.65 & 0.65 & $0.636 \pm 0.068^{\dagger}$ & $0.686 \pm 0.021^{\dagger}$ & 0.73 & 0.93 \\
\hline$\Xi$ & - & -0.21 & -0.22 & -0.22 & $-0.277 \pm 0.034$ & $-0.299 \pm 0.014^{\ddagger}$ & $-0.23^{\ddagger}$ & -0.32 \\
\hline$\Delta$ & - & -4.48 & -4.47 & -4.30 & - & - & $\sim-4.5$ & -6.00 \\
\hline$\Sigma^{*}$ & - & -1.06 & -1.06 & -1.00 & - & - & - & -1.41 \\
\hline$\Xi^{*}$ & - & -0.75 & -0.75 & -0.70 & - & - & - & -1.00 \\
\hline
\end{tabular}

$\dagger$ Due to another definition of $g_{A}^{\Sigma}$ this numerical value is different by a $\sqrt{2}$ from the one quoted in the original paper.

$\ddagger$ Due to another definition of $g_{A}^{\Xi}$ this value has a sign opposite to the one in the original paper.

the psGBE RCQM, interestingly, it has recently been found 28] that also the $\pi N N$ coupling constant turns out to be too small, namely $\frac{f_{\pi N N}^{2}}{4 \pi}=0.0691$, as compared to the phenomenological value of about 0.075 [35]. It remains to be clarified if in case of the RCQMs these undershootings of both the $g_{A}^{N}$ and $f_{\pi N N}^{2}$, which are related by the Goldberger-Treiman relation, have to be interpreted as lacking $\pi$-dressing effects.

In the last column of Table $\Pi$ we quote also the nonrelativistic limit of the prediction by the EGBE RCQM (i.e. for the limit $\kappa \rightarrow 1$ in Eq. (11)). It deviates grossly from the relativistic result, indicating that a consideration of axial charges within a nonrelativistic approach is unreliable. This conclusion is further substantiated by considering the axial charges of $N$ resonances, for which indeed no experimental data are available but latticeQCD results have recently been produced. While the relativistic predictions of especially the EGBE RCQM agree very well with the lattice-QCD data in case of both the $N(1535)$ and $N(1650)$ resonances, the nonrelativistic limits deviate here too [18].

For the $g_{A}^{B}$ of the octet and decuplet ground states the RCQMs yield very similar results. While the predictions of the psGBE and the EGBE are essentially the same, differences occur only for the OGE RCQM, but they remain within at most $\sim 6 \%$. In case of the octet states $\Sigma$ and $\Xi$ we can also compare to lattice-QCD as well as $\chi \mathrm{PT}$ results. The comparison of the RCQM predictions to the former is quite satisfying, as the figures agree rather well. Except for $g_{A}^{\Sigma}$ practically the same is true with regard to the $\chi \mathrm{PT}$ results of JT. Again, the results from the nonrelativistic limit of the EGBE RCQM fall short; as in the case of the $N$ the corresponding values are always bigger (in absolute value) than all of the other results.

For the decuplet ground states $\Delta, \Sigma^{*}$, and $\Xi^{*}$ there are neither experimental data nor lattice-QCD results. Only for the $\Delta$ we may compare with a $\chi \mathrm{PT}$ prediction, showing again a striking similarity. For the other cases of $\Sigma^{*}$ and $\Xi^{*}$ we have here produced first predictions and one has still to await results from other approaches.

TABLE II: Mass eigenvalues and axial charges $g_{A}^{N}$ of the $N$ ground state and the low-lying $N$ resonances as predicted by the EGBE, the psGBE, and the OGE RCQMs.

\begin{tabular}{llccccccc}
\hline \hline & & \multicolumn{2}{c}{ EGBE } & \multicolumn{2}{c}{ psGBE } & \multicolumn{2}{c}{ OGE } \\
\hline State & $J^{p}$ & Mass & $g_{A}$ & Mass & $g_{A}$ & Mass & $g_{A}$ \\
\hline & & & & & & & \\
$N(939)$ & $\frac{1}{2}^{+}$ & 939 & 1.15 & 939 & 1.15 & 939 & 1.11 \\
$N(1440)$ & $\frac{1}{2}^{+}$ & 1464 & 1.16 & 1459 & 1.13 & 1578 & 1.10 \\
$N(1520)$ & $\frac{3}{2}^{-}$ & 1524 & -0.64 & 1519 & -0.21 & 1520 & -0.15 \\
$N(1535)$ & $\frac{1}{2}^{-}$ & 1498 & 0.02 & 1519 & 0.09 & 1520 & 0.13 \\
$N(1650)$ & $\frac{1}{2}^{-}$ & 1581 & 0.51 & 1647 & 0.46 & 1690 & 0.44 \\
$N(1700)$ & $\frac{3}{2}^{-}$ & 1608 & -0.10 & 1647 & -0.50 & 1690 & -0.47 \\
$N(1710)$ & $\frac{1}{2}^{+}$ & 1757 & 0.35 & 1776 & 0.37 & 1860 & 0.32 \\
$N(1720)$ & $\frac{3}{2}^{+}$ & 1746 & 0.35 & 1728 & 0.34 & 1858 & 0.25 \\
$N(1675)$ & $\frac{5}{2}^{-}$ & 1676 & 0.84 & 1647 & 0.83 & 1690 & 0.80 \\
$N(1680)$ & $\frac{5}{2}^{+}$ & 1689 & 0.89 & 1728 & 0.83 & 1858 & 0.70 \\
\hline \hline
\end{tabular}

Next we come to discuss the axial charges of nucleon and other baryon resonances. As mentioned in the Introduction, especially the nucleon resonances have recently attracted interest, mainly because the issue of chiralsymmetry restoration higher in the baryon spectra has been raised [15, 16] and because first lattice-QCD calculations have been performed [17]. Certainly the results of the latter have still to be taken with care, as they correspond to relatively high quark masses. For the case of the nucleon we have presented resonance axial charges from RCQMs already in a previous paper [18]; for completeness we repeat them here in Table II. While for details we refer to ref. [18], we mention as the main characterization of these results that 
i) the RCQM predictions perfectly agree with the latticeQCD results for the $N(1535)$ and $N(1650)$ resonances, i.e. in the two cases for which lattice-QCD calculations have so far become available,

ii) the small, practically vanishing, $g_{A}$ of $N(1535)$ can be reproduced with $\{Q Q Q\}$ configurations alone,

iii) the predictions of different RCQMs are generally very similar except for the $J^{P}=\frac{3}{2}^{-}$resonances $N(1520)$ and $N(1700)$,

$i v)$ a relativistic description is necessary and a simple $S U(6) \times O(3)$ nonrelativistic quark model is not reliable, and

$v$ ) there is no tendency of the axial charges of high-lying parity partners to assume particularly small values.

TABLE III: Same as Table IIbut for the octet $\Sigma$ and decuplet $\Sigma^{*}$ states.

\begin{tabular}{lllllllll}
\hline \hline & & \multicolumn{2}{c}{ EGBE } & \multicolumn{2}{c}{ psGBE } & \multicolumn{2}{c}{ OGE } \\
\hline State & $J^{p}$ & Mass & $g_{A}$ & Mass & $g_{A}$ & Mass & $g_{A}$ \\
\hline$\Sigma(1193)$ & $\frac{1}{2}^{+}$ & 1194 & 0.65 & 1182 & 0.65 & 1121 & 0.65 \\
$\Sigma(1560)$ & $\frac{1}{2}^{-}$ & 1672 & -0.15 & 1678 & -0.07 & 1655 & 0.01 \\
$\Sigma(1620)$ & $\frac{1}{2}^{-}$ & 1740 & 0.62 & 1736 & 0.58 & 1770 & 0.54 \\
$\Sigma(1660)$ & $\frac{1}{2}^{+}$ & 1664 & 0.69 & 1619 & 0.64 & 1755 & 0.64 \\
$\Sigma(1670)$ & $\frac{3}{2}^{-}$ & 1681 & -0.92 & 1678 & -0.48 & 1655 & -0.24 \\
$\Sigma(1775)$ & $\frac{5}{2}^{-}$ & 1765 & 1.06 & 1736 & 1.03 & 1770 & 0.97 \\
$\Sigma(1880)$ & $\frac{1}{2}^{+}$ & 1903 & 0.38 & 1912 & 0.42 & 1980 & 0.17 \\
$\Sigma(1940)$ & $\frac{3}{2}^{-}$ & 1725 & -0.45 & 1736 & -0.83 & 1770 & -0.78 \\
\hline$\Sigma^{*}(1385)$ & $\frac{3}{2}^{+}$ & 1365 & -1.06 & 1389 & -1.06 & 1311 & -1.00 \\
$\Sigma^{*}(1690)$ & $\frac{3}{2}^{+}$ & 1812 & -1.05 & 1865 & -1.03 & 1932 & -0.99 \\
$\Sigma^{*}(1750)$ & $\frac{1}{2}^{-}$ & 1761 & -0.08 & 1759 & -0.13 & 1718 & -0.18 \\
\hline \hline
\end{tabular}

The RCQM predictions for the axial charges of the octet $\Sigma$ and decuplet $\Sigma^{*}$ resonances are quoted in $\mathrm{Ta}$ ble III. The gross pattern of the results is like the one of the $N$ resonances. First of all the different RCQMs yield similar values for the axial charges except for the cases of $\Sigma(1670)$ and $\Sigma(1940)$, which are again $J^{P}=\frac{3}{2}^{-}$ resonances and are to be assigned to the same octets as $N(1520)$ and $N(1700)$, respectively, according to a recent identification of baryon resonances [36] (see also [37]). For both $\Sigma(1670)$ and $\Sigma(1940)$ the differences among the predictions prevail also in the comparison between the EGBE and the psGBE RCQMs hinting to considerable influences from tensor and/or spin-orbit forces, just as in the corresponding two $N$ resonances. All of the RCQMs produce very small values for the axial charges of $\Sigma(1560)$. Notably, this state falls into the same octet as $N(1535)$ [36, 37], whose $g_{A}$ is also extremely small (see Table II). A similar small axial charge is found for the decuplet $\Sigma^{*}(1750)$. In general, however, we do not ob- serve the axial charges to become small as we go up to higher resonances.

The results for the axial charges of the octet $\Xi$ and decuplet $\Xi^{*}$ resonances are collected in Table IV] Here, all the RCQMs produce similar predictions, where the axial charges of the octet resonances remain rather small with values ranging from -0.2 to -0.4 .

TABLE IV: Same as Table $\amalg$ but for the octet $\Xi$ and decuplet $\Xi^{*}$ states..

\begin{tabular}{llcccccc}
\hline \hline & \multicolumn{2}{c}{ EGBE } & \multicolumn{2}{c}{ psGBE } & \multicolumn{2}{c}{ OGE } \\
\hline State & $J^{p}$ & Mass & $g_{A}$ & Mass & $g_{A}$ & Mass & $g_{A}$ \\
\hline$\Xi(1318)$ & $\frac{1}{2}^{+}$ & 1355 & -0.21 & 1348 & -0.22 & 1193 & -0.22 \\
$\Xi(1690)$ & $\frac{1}{2}^{+}$ & 1813 & -0.23 & 1806 & -0.22 & 1826 & -0.22 \\
$\Xi(1820)$ & $\frac{3}{2}^{-}$ & 1807 & -0.38 & 1792 & -0.40 & 1751 & -0.23 \\
\hline$\Xi^{*}(1530)$ & $\frac{3}{2}^{+}$ & 1512 & -0.75 & 1528 & -0.75 & 1392 & -0.70 \\
\hline \hline
\end{tabular}

Finally, in Table $\nabla$ the axial charges of the $\Delta$ resonances are given. Again, all of the RCQMs yield similar predictions. Only it is remarkable that the axial charges especially of the $J^{P}=\frac{3}{2}^{+}$states are rather big in absolute value. If we consider the $\Delta(1232)$ ground state, its $g_{A}$ is at least three times larger than the one of the $N$ ground state. Remarkably a ratio of about the same size has recently been found between the $\pi N \Delta$ and the $\pi N N$ strong coupling constants 33. The smallest $g_{A}$ is found for $\Delta(1620)$. It should be noted that it falls into the same decuplet as the $\Sigma^{*}(1750)$, whose $g_{A}$ was also seen as the smallest among the $\Sigma^{*}$ resonances (cf. Table III).

TABLE V: Same as Table II but for the $\Delta$ states.

\begin{tabular}{llllllll}
\hline \hline & & \multicolumn{2}{c}{ EGBE } & \multicolumn{2}{c}{ psGBE } & \multicolumn{2}{c}{ OGE } \\
\hline State & $J^{p}$ & Mass & $g_{A}$ & Mass & $g_{A}$ & Mass & $g_{A}$ \\
\hline & & & & & & & \\
$\Delta(1232)$ & $\frac{3}{2}^{+}$ & 1231 & -4.48 & 1240 & -4.47 & 1231 & -4.30 \\
$\Delta(1600)$ & $\frac{3}{2}^{+}$ & 1686 & -4.41 & 1718 & -4.33 & 1855 & -4.20 \\
$\Delta(1620)$ & $\frac{1}{2}^{-}$ & 1640 & -0.76 & 1642 & -0.75 & 1621 & -0.74 \\
$\Delta(1700)$ & $\frac{3}{2}^{-}$ & 1639 & -1.68 & 1642 & -1.66 & 1621 & -1.58 \\
\hline \hline
\end{tabular}

\section{CONCLUSIONS}

We have presented results from a comprehensive and consistent study of axial charges $g_{A}^{B}$ of octet and decuplet baryon ground and resonant states with RCQMs. The dynamical models differ mainly with regard to their hyperfine $Q-Q$ interactions, which stem from OGE and psGBE as well as EGBE. Whenever a comparison is possible with either experimental data or established theoretical results (especially from lattice $\mathrm{QCD}$ and $\chi \mathrm{PT}$ ), 
the RCQM predictions turn out to be quite reasonable. The values deduced from a nonrelativistic approximation in general differ grossly, indicating that a relativistic approach to the axial charges is mandatory. The RCQMs considered here rely on $\{Q Q Q\}$ configurations only. Nevertheless, the $g_{A}^{B}$ results never fall short but rather produce a consistent picture. Already for the ground states one finds a scatter of $g_{A}^{B}$ values from small to large. Through the Goldberger-Treiman relation one may thus expect smaller or larger $\pi$-dressing effects depending on the baryon state. Particularly big are the axial charges of the $\delta$ ground and first excited states, much in congruency with the relatively large $\pi N \Delta$ coupling constant. The axial charges of some baryon resonances are rather sensitive to tensor and/or spin-orbit forces in the hyperfine interaction. These resonances fall into the same flavor multiplets. In general the pattern observed from the predictions for $g_{A}^{B}$ is congruent with the classification of baryons into flavor multiplets as found recently. From the RCQMs predictions presented here, no particular trend is observed for the axial charges of $N$ and other baryon resonances to become small, when the excitation energy is increased. Certainly, the consideration of baryon axial charges remains an exciting field, and one is eager to see additional experimental data as well as more theoretical results from different approaches to QCD.

\section{Acknowledgments}

The authors are grateful to L.Ya. Glozman for valuable incentives regarding specific aspects of this work and to the Graz lattice-QCD group for several clarifying discussions about respective calculations. This work was supported by the Austrian Science Fund, FWF, through the Doctoral Program on Hadrons in Vacuum, Nuclei, and Stars (FWF DK W1203-N08).

\section{Appendix: Rarita-Schwinger spinors}

For the $J=\frac{1}{2}, \frac{3}{2}$, and $\frac{5}{2}$ baryons with four-momentum $P$ and energy $E=\sqrt{M^{2}+\vec{P}^{2}}$ we employ Dirac and Rarita-Schwinger spinors, similar as in ref. [38], as follows:

- $J=\frac{1}{2}, U\left(P, J_{3}= \pm \frac{1}{2}\right)$ :

$$
U\left(P, \frac{1}{2}\right)=\left(\begin{array}{c}
\sqrt{E+M} \\
0 \\
\frac{\vec{\sigma} \cdot \vec{P}}{\sqrt{E+M}} \\
0
\end{array}\right), \quad U\left(P,-\frac{1}{2}\right)=\left(\begin{array}{c}
0 \\
\sqrt{E+M} \\
0 \\
\frac{\vec{\sigma} \cdot \vec{P}}{\sqrt{E+M}}
\end{array}\right)
$$

where $\vec{\sigma}$ are the Pauli matrices. These Dirac spinors are normalized as

$$
\bar{U}\left(P, J_{3}^{\prime}\right) U\left(P, J_{3}\right)=\delta_{J_{3}^{\prime}, J_{3}} 2 M
$$

- $J=\frac{3}{2}, U^{\mu}\left(P, J_{3}= \pm \frac{1}{2}, \pm \frac{3}{2}\right)$ :

$$
\begin{aligned}
U^{\mu}\left(P, \frac{3}{2}\right) & =e_{+}^{\mu}(P) U\left(P, \frac{1}{2}\right), \\
U^{\mu}\left(P, \frac{1}{2}\right) & =\sqrt{\frac{2}{3}} e_{0}^{\mu}(P) U\left(P, \frac{1}{2}\right)+\sqrt{\frac{1}{3}} e_{+}^{\mu}(P) U\left(P,-\frac{1}{2}\right), \\
U^{\mu}\left(P,-\frac{1}{2}\right) & =\sqrt{\frac{1}{3}} e_{-}^{\mu}(P) U\left(P, \frac{1}{2}\right)+\sqrt{\frac{2}{3}} e_{0}^{\mu}(P) U\left(P,-\frac{1}{2}\right), \\
U^{\mu}\left(P,-\frac{3}{2}\right) & =e_{-}^{\mu}(P) U\left(P,-\frac{1}{2}\right) .
\end{aligned}
$$


- $J=\frac{5}{2}, U^{\mu \nu}\left(P, J_{3}= \pm \frac{1}{2}, \pm \frac{3}{2}, \pm \frac{5}{2}\right)$ :

$$
\begin{aligned}
U^{\mu \nu}\left(P, \frac{5}{2}\right)= & e_{+}^{\mu} e_{+}^{\nu} U\left(P, \frac{1}{2}\right), \\
U^{\mu \nu}\left(P, \frac{3}{2}\right)= & \sqrt{\frac{2}{5}} e_{+}^{\mu} e_{0}^{\nu} U\left(P, \frac{1}{2}\right)+\sqrt{\frac{1}{5}} e_{+}^{\mu} e_{+}^{\nu} U\left(P,-\frac{1}{2}\right)+\sqrt{\frac{2}{5}} e_{0}^{\mu} e_{+}^{\nu} U\left(P, \frac{1}{2}\right), \\
U^{\mu \nu}\left(P, \frac{1}{2}\right)= & \sqrt{\frac{1}{10}} e_{+}^{\mu} e_{-}^{\nu} U\left(P, \frac{1}{2}\right)+\sqrt{\frac{1}{5}} e_{+}^{\mu} e_{0}^{\nu} U\left(P,-\frac{1}{2}\right)+\sqrt{\frac{2}{5}} e_{0}^{\mu} e_{0}^{\nu} U\left(P, \frac{1}{2}\right) \\
& +\sqrt{\frac{1}{5}} e_{0}^{\mu} e_{+}^{\nu} U\left(P,-\frac{1}{2}\right)+\sqrt{\frac{1}{10}} e_{-}^{\mu} e_{+}^{\nu} U\left(P, \frac{1}{2}\right), \\
U^{\mu \nu}\left(P,-\frac{1}{2}\right)= & \sqrt{\frac{1}{10}} e_{+}^{\mu} e_{-}^{\nu} U\left(P,-\frac{1}{2}\right)+\sqrt{\frac{1}{5}} e_{0}^{\mu} e_{-}^{\nu} U\left(P, \frac{1}{2}\right)+\sqrt{\frac{2}{5}} e_{0}^{\mu} e_{0}^{\nu} U\left(P,-\frac{1}{2}\right) \\
& +\sqrt{\frac{1}{5}} e_{-}^{\mu} e_{0}^{\nu} U\left(P, \frac{1}{2}\right)+\sqrt{\frac{1}{10}} e_{-}^{\mu} e_{+}^{\nu} U\left(P,-\frac{1}{2}\right), \\
U^{\mu \nu}\left(P,-\frac{3}{2}\right)= & \sqrt{\frac{2}{5}} e_{0}^{\mu} e_{-}^{\nu} U\left(P,-\frac{1}{2}\right)+\sqrt{\frac{1}{5}} e_{-}^{\mu} e_{-}^{\nu} U\left(P,-\frac{1}{2}\right)+\sqrt{\frac{2}{5}} e_{-}^{\mu} e_{0}^{\nu} U\left(P,-\frac{1}{2}\right), \\
U^{\mu \nu}\left(P,-\frac{5}{2}\right)= & e_{-}^{\mu} e_{-}^{\nu} U\left(P,-\frac{1}{2}\right) .
\end{aligned}
$$

In the latter equation we have suppressed the arguments in the polarization vectors $e_{\lambda}^{\mu}(P)$ defined by

$$
e_{\lambda}^{\mu}(P)=\left(\frac{\hat{e}_{\lambda} \cdot \vec{P}}{M}, \hat{e}_{\lambda}+\frac{\left(\hat{e}_{\lambda} \cdot \vec{P}\right) \vec{P}}{M(E+M)}\right),
$$

where for $\lambda=+, 0,-$ the unit vectors $\hat{e}_{\lambda}$ are written as

$$
\hat{e}_{+}=-\frac{1}{\sqrt{2}}(1, i, 0), \hat{e}_{0}=(0,0,1), \hat{e}_{-}=\frac{1}{\sqrt{2}}(1,-i, 0) .
$$

[1] M. L. Goldberger and S. B. Treiman, Phys. Rev. 110, 1178 (1958).

[2] C. Amsler et al. [Particle Data Group], Phys. Lett. B 667, 1 (2008).

[3] S. L. Adler, Phys. Rev. Lett. 14, 1051 (1965).

[4] W. I. Weisberger, Phys. Rev. Lett. 14, 1047 (1965).

[5] D. Renner, PoS(LATTICE 2009), 18 (2009).

[6] H. W. Lin and K. Orginos, Phys. Rev. D 79, 034507 (2009).

[7] G. Erkol, M. Oka, and T. T. Takahashi, Phys. Lett. B 686, 36 (2010).

[8] G. Engel, C. Gattringer, L. Y. Glozman, C. B. Lang, M. Limmer, D. Mohler, and A. Schäfer, PoS(LATTICE 2009), 135 (2009); arXiv:0910.4190

[9] V. Bernard, Prog. Part. Nucl. Phys. 60, 82 (2008).

[10] F. J. Jiang and B. C. Tiburzi, Phys. Rev. D 80, 077501 (2009).

[11] J. C. Nacher, A. Parreno, E. Oset, A. Ramos, A. Hosaka, and M. Oka, Nucl. Phys. A 678, 187 (2000).

[12] L. Y. Glozman, M. Radici, R. F. Wagenbrunn, S. Boffi, W. Klink, and W. Plessas, Phys. Lett. B 516, 183 (2001).

[13] S. Boffi, L. Y. Glozman, W. Klink, W. Plessas, M. Radici, and R. F. Wagenbrunn, Eur. Phys. J. A 14, 17 (2002).

[14] D. Merten, U. Löring, K. Kretzschmar, B. Metsch, and H. R. Petry, Eur. Phys. J. A 14, 477 (2002).

[15] L. Y. Glozman, Phys. Rep. 444, 1 (2007).

[16] L. Y. Glozman and A. V. Nefediev, Nucl. Phys. A 807, 38 (2008).

[17] T. T. Takahashi and T. Kunihiro, Phys. Rev. D 78, 011503 (2008).

[18] Ki-Seok Choi, W. Plessas, and R .F. Wagenbrunn, Phys. Rev. C 81, 028201 (2010).

[19] C. S. An and D. O. Riska, Eur. Phys. J. A 37, 263 (2008).

[20] S. G. Yuan, C. S. An, and J. He, arXiv:0908.2333.

[21] J. M. Gaillard and G. Sauvage, Ann. Rev. Nucl. Part. Sci. 34, 351 (1984).

[22] K. Dannbom, L. Y. Glozman, C. Helminen, and D. O. Riska, Nucl. Phys. A 616, 555 (1997).

[23] J. J. de Swart, Rev. Mod. Phys. 35, 916 (1963) [Erratumibid. 37, 326 (1965)].

[24] L. Theussl, R. F. Wagenbrunn, B. Desplanques, and W. Plessas, Eur. Phys. J. A 12, 91 (2001).

[25] L. Y. Glozman, Z. Papp, W. Plessas, K. Varga, and R. F. Wagenbrunn, Phys. Rev. C 57, 3406 (1998). 
[26] L. Y. Glozman, W. Plessas, K. Varga, and R. F. Wagenbrunn, Phys. Rev. D 58, 094030 (1998).

[27] K. Glantschnig, R. Kainhofer, W. Plessas, B. Sengl, and R. F. Wagenbrunn, Eur. Phys. J. A 23, 507 (2005).

[28] T. Melde, L. Canton, W. Plessas, and R. F. Wagenbrunn, Eur. Phys. J. A 25, 97 (2005).

[29] R. F. Wagenbrunn, S. Boffi, W. Klink, W. Plessas, and M. Radici, Phys. Lett. B 511, 33 (2001).

[30] K. Berger, R. F. Wagenbrunn, and W. Plessas, Phys. Rev. D 70, 094027 (2004).

[31] T. Melde, K. Berger, L. Canton, W. Plessas, and R. F. Wagenbrunn, Phys. Rev. D 76, 074020 (2007).

[32] W. Rarita and J. Schwinger, Phys. Rev. 60, 61 (1941).

[33] T. Melde, L. Canton, and W. Plessas, Phys. Rev. Lett.
102, 132002 (2009).

[34] F. J. Jiang and B. C. Tiburzi, Phys. Rev. D 78, 017504 (2008).

[35] D. V. Bugg, Eur. Phys. J. C 33, 505 (2004).

[36] T. Melde, W. Plessas, and B. Sengl, Phys. Rev. D 77, 114002 (2008).

[37] Online version of the review on the Quark Model by the Particle Data Group available from http://pdg.lbl.gov/2009/reviews/rpp2009-rev-quark-model.pdf (print version to appear in 2010).

[38] K. S. Choi, S. I. Nam, A. Hosaka, and H. C. Kim, J. Phys. G 36, 015008 (2009). 